\title{
Attainable values for upper porosities of measures.
}

\author{
M. Eugenia Mera. Manuel Morán.
}

\begin{abstract}
.
We consider two definitions of upper porosity of measures and we prove that the first one only can take the values 0 and $\frac{1}{2}$ and the second one the values $0, \frac{1}{2}$ or 1 .

Keywords and Phrases: Doubling Condition, Porosity of Sets, Porosity of Measures, Tangent Measures.

Mathematical Subject Classification (1991) 28A05, 28A12.

\section{Results.}

In this paper we introduce two definitions of upper porosity of a measure (see Definitions 1.3 and 1.5) which range from 0 to $\frac{1}{2}$ and from 0 to 1 respectively, and prove (Theorem 1.8 and Corollary 1.9) that actually the first porosity only can take the extreme values 0 or $\frac{1}{2}$, and the second one takes either the value 0 or the values $\frac{1}{2}$ or 1 . The other main result of this paper (see Theorem 1.2, Corollary 1.4 and Proposition 1.6) says that any measure $\mu$ which does not satisfy the doubling condition $\mu$-a.e. has a maximal porosity.
\end{abstract}

\subsection{Porosities of sets and the doubling condition.}

Let $B(x, r)$ be the closed ball with center $x \in \mathrm{IR}^{n}$ and radius $r$. For $A \subset \mathrm{IR}^{n}$, $x \in \operatorname{IR}^{n}$ and $r>0$, let

$$
p(A, x, r)=\sup \left\{\rho: B(z, \rho) \subset B(x, r) \backslash A \text { for some } z \in \mathrm{IR}^{n}\right\},
$$




$$
\begin{aligned}
& \bar{p}(A, x)=\lim \sup _{r \downarrow 0} \frac{p(A, x, r)}{r} \text { and } \\
& \underline{p}(A, x)=\liminf \sin _{r \downarrow 0} \frac{p(A, x, r)}{r} .
\end{aligned}
$$

For $x \in A, p(A, x, r)$ takes a value in between 0 and $r / 2$, so $\bar{p}(A, x)$ and $\underline{p}(A, x)$ take values in between 0 and $\frac{1}{2}$.

The upper and lower porosity of a set $A$ are given by

$$
\bar{p}(A)=\inf \{\bar{p}(A, x): x \in A\} \text { and } \underline{p}(A)=\inf \{\underline{p}(A, x): x \in A\}
$$

respectively. The set $A$ is said to be porous if $\bar{p}(A)>0$ and very porous if $\underline{p}(A)>0$. The set $A$ is said to be strongly porous if $\bar{p}(A)=\frac{1}{2}$ and strongly very porous if $\underline{p}(A)=\frac{1}{2}$. The set $A$ is said to be $\sigma$-porous ( $\sigma$-very porous, $\sigma$-strongly porous, $\sigma$-strongly very porous) if $A$ is a countable union of porous (very porous, strongly porous, strongly very porous) sets. Results on porous sets connected with problems in analysis can be seen in [9] and [10], and results on Hausdorff dimension of very porous sets can be found in [5] and [8].

The doubling condition is usually imposed in problems of harmonic analysis, Vitali coverings theorems and tangent measures theory ([1],[2],[4] and [5]).

A probability measure $\mu$ on $\operatorname{IR}^{n}$ satisfies the doubling condition at a point $a \in \operatorname{IR}^{n}$ if

$$
\lim \sup _{r \downarrow 0} \frac{\mu(B(a, 2 r))}{\mu(B(a, r))}<\infty .
$$

\subsection{Main results.}

We begin studying the Radon probability measures $\mu$ on $\operatorname{IR}^{n}$ which do not satisfy the doubling condition $\mu$-a.e. We prove (see Theorem 1.2) that any Radon probability measure $\mu$ gives two alternative decompositions of $\operatorname{IR}^{n}$ into three sets:

- the set where the doubling condition holds, a set with arbitrary small $\mu$ measure and a strongly porous set. This last set is contained in a very sparse set defined as an intersection of disjointed unions of annuli of width tending to zero (see Lemma 1.1 below).

- the set of points where the doubling condition holds, a set of null $\mu$-measure and a $\sigma$-strongly porous set. 
The following lemma describes the geometry of the set of points where a measure does not satisfy the doubling condition.

Lemma 1.1. Let $\mu$ be a Radon probability measure on $\mathrm{IR}^{n}$ and let $A$ be the set of points where $\mu$ does not satisfy the doubling condition. Let $\left\{\lambda_{i}\right\}$ be a sequence of real numbers such that $\lim _{i \rightarrow \infty} \lambda_{i}=1$ and $0<\lambda_{i}<1, i \in$ IN. Then for any $\varepsilon>0$, there exist a family $\left\{x_{i, j}\right\}_{i, j \in \mathrm{IN}}$ of points in $A$ and a family $\left\{r_{i, j}\right\}_{i, j \in \mathrm{IN}}$ of radii, with $r_{i, j}<1 / i$ for all $j \in \mathrm{IN}$, such that

$$
\mu\left(A \backslash\left(\bigcap_{i=1}^{\infty} \bigcup_{j=1}^{\infty} W_{i, j}\right)\right) \leq \varepsilon
$$

where $W_{i, j}:=B\left(x_{i, j}, r_{i, j}\right) \backslash B\left(x_{i, j}, \lambda_{i} r_{i, j}\right)$, and for any $i \in$ IN the balls in the family $\left\{B\left(x_{i, j}, r_{i, j}\right)\right\}_{j \in \mathrm{IN}}$ are disjointed balls.

This result gives a strong indication that the measures which do not satisfy the doubling condition are exceptional. In particular we conjecture that an ergodic measure invariant for a smooth hyperbolic dynamical system in a $n$-dimensional manifold must satisfy the doubling condition. We have been unable to prove this conjecture from Lemma 1.1, which, however, gives easily the following result relating porosity to doubling condition.

Theorem 1.2. Let $\mu$ be a Radon probability measure on $\operatorname{IR}^{n}$ and let $A$ be the set of points where $\mu$ does not satisfy the doubling condition. The following statements hold.

i) For all $\varepsilon>0$, there is a strongly porous subset $A^{*}$ of $A$ such that $\mu\left(A \backslash A^{*}\right) \leq \varepsilon$. ii) There exists a $\sigma$-strongly porous subset $C$ of $A$ such that $\mu(A)=\mu(C)$.

This theorem suggests the following definitions of porosity of a measure.

Definition 1.3. Let $\mu$ be a measure over $\mathrm{IR}^{n}$. We define the upper and lower porosity of $\mu$ as

$$
\bar{p}(\mu)=\sup \left\{\bar{p}(A): A \subset \operatorname{IR}^{n} \text { with } \mu(A)>0\right\}
$$

and

$$
\underline{p}(\mu)=\sup \left\{\underline{p}(A): A \subset \operatorname{IR}^{n} \text { with } \mu(A)>0\right\}
$$

respectively. We say that $\mu$ is a porous measure if $\bar{p}(\mu)>0$ and a very porous measure if $p(\mu)>0$. The notions of strongly porous and very strongly porous measures are defined in the obvious way. 
Corollary 1.4. Let $\mu$ be a Radon probability measure on $\mathrm{IR}^{n}$ which does not satisfy the doubling condition $\mu$-a.e. Then $\bar{p}(\mu)=\frac{1}{2}$.

We will use this corollary in proving that any porous measure is a strongly porous measure (see Theorem 1.8).

We now introduce another definition of upper porosity of a measure $\mu$ which is equivalent, when the measure $\mu$ satisfies the doubling condition $\mu$-a.e., to that given in definition 1.3. We use this equivalence in the proof of Theorem 1.8.

Definition 1.5. The upper porosity $\overline{p o r}(\mu)$ of $\mu$ is given by

$$
\overline{\operatorname{por}}(\mu):=\inf \left\{s: \overline{\operatorname{por}}(\mu, x) \leq s, \mu \text {-a.e } x \in \operatorname{IR}^{n}\right\}
$$

where

$$
\overline{\operatorname{por}}(\mu, x):=\lim _{\varepsilon \downarrow 0} \limsup _{r \downarrow 0} \operatorname{por}(\mu, x, r, \varepsilon)
$$

is the upper porosity of $\mu$ at $x$ and

$$
\begin{aligned}
\operatorname{por}(\mu, x, r, \varepsilon):=\sup \{\rho: & \text { there is a } z \in \operatorname{IR}^{n} \text { such that } B(z, \rho r) \subset B(x, r) \\
& \text { and } \mu(B(z, \rho r)) \leq \varepsilon \mu(B(x, r))\} .
\end{aligned}
$$

Notice that $\overline{p o r}(\mu)$ ranges from 0 to 1 . This is the version for the upper porosity of the following definition of lower porosity $\operatorname{por}(\mu)$ given by J-P. Eckmann, E. Järvenpää and M. Järvenpää in [3]:

$$
\underline{\operatorname{por}}(\mu)=\inf \left\{s: \underline{\operatorname{por}}(\mu, x) \leq s, \mu \text {-a.e. } x \in \operatorname{IR}^{n}\right\},
$$

where

$$
\underline{\operatorname{por}}(\mu, x):=\lim _{\varepsilon \downarrow 0} \liminf _{r \downarrow 0} \operatorname{por}(\mu, x, r, \varepsilon),
$$

is the lower porosity of $\mu$ at $x$.

They prove that $\operatorname{por}(\mu) \leq \underline{p}(\mu)$ holds for any Radon probability measure $\mu$, and if $\mu$ satisfies the doubling condition $\mu$-a.e. then $\operatorname{por}(\mu)=p(\mu)$, but $\operatorname{por}(\mu)>$ $p(\mu)$ may occur if the doubling condition fails to hold $\mu$-a.e. ([3], example 4). Obvious changes in the proof of these facts give the corresponding results for the upper porosities of the measure, that is $\bar{p}(\mu) \leq \overline{p o r}(\mu)$ for any Radon probability measure $\mu$, and if $\mu$ satisfies the doubling condition $\mu$-a.e. then $\bar{p}(\mu) \geq \overline{p o r}(\mu)$, and hence $\overline{p o r}(\mu)=\bar{p}(\mu)$.

Notice that if $\mu$ does not satisfy the doubling condition $\overline{p o r}(\mu) \geq \bar{p}(\mu)=\frac{1}{2}$ holds. We prove that in this case $\overline{p o r}(\mu)=1$. 
Proposition 1.6. Let $\mu$ be a Radon probability measure on $\mathrm{IR}^{n}$ which does not satisfy the doubling condition $\mu$-a.e. Then $\overline{\operatorname{por}}(\mu)=1$.

The next lemma characterizes strongly porous measures in terms of their tangent measures.

Tangent measures, introduced by Preiss ([7]), have turned out to be a powerful tool for the study of the local behaviour of measures. Given a locally finite Borel measure $\mu$ over $\mathrm{IR}^{n}$, the measure $\nu$ is a tangent measure of $\mu$ at a point $a$ if it is a non null locally finite Borel measure and there are sequences $\left\{c_{i}\right\}$ and $\left\{r_{i}\right\}$ of positive numbers such that $\left\{r_{i}\right\} \downarrow 0$ and

$$
c_{i} T_{a, r_{i} \#} \mu \stackrel{w}{\rightarrow} \nu
$$

holds, where $T_{a, r_{i}}$ are the homotheties given by $T_{a, r_{i}}(x)=\frac{x-a}{r_{i}}, T_{a, r_{i} \#} \mu$ is the measure induced by $T_{a, r_{i}}$, (i.e. $T_{a, r_{i} \#} \mu(A)=\mu\left(a+r_{i} A\right), A \subset \mathrm{IR}^{n}$ ) and $\stackrel{w}{\rightarrow}$ denotes the weak convergence of measures. The set of all such tangent measures is denoted by $\operatorname{Tan}(\mu, a)$ and the support of the measure $\mu$ is denoted by $\operatorname{spt}(\mu)$.

Lemma 1.7. Let $\mu$ be a Radon probability measure on $I R^{n}$ satisfying the doubling condition $\mu$-a.e. Let

$$
B:=\left\{a \in \operatorname{IR}^{n}: \text { there is } \nu \in \operatorname{Tan}(\mu, a) \text { such that } \operatorname{spt}(\nu) \neq \operatorname{IR}^{n}\right\} .
$$

Then

$$
\bar{p}(\mu)=\frac{1}{2} \Longleftrightarrow \mu(B)>0
$$

From this lemma easily follows the main result of this paper:

Theorem 1.8. Let $\mu$ be a Radon probability measure on $\operatorname{IR}^{n}$. Then $\bar{p}(\mu)$ is either 0 or $\frac{1}{2}$.

Corollary 1.9. Let $\mu$ be a Radon probability measure on $\operatorname{IR}^{n}$. Then $\overline{p o r}(\mu)$ is $0, \frac{1}{2}$ or 1 .

We only can obtain the lower bound $\frac{1}{4}$ for the porosity of subsets arbitrarily close in measure to a given porous set, although it seems likely that this bound can be improved to $\frac{1}{2}$. 
Theorem 1.10. Let $\mu$ be a Radon probability measure on $I R^{n}$ which satisfies the doubling condition $\mu$-a.e. and let $A \subset \mathrm{IR}^{n}$. If $\bar{p}(A)>0$ then for any $\varepsilon$, $0<\varepsilon<\mu(A)$, there is a set $A^{*} \subset A$ such that $\mu\left(A \backslash A^{*}\right) \leq \varepsilon$ and $\bar{p}\left(A^{*}\right) \geq \frac{1}{4}$.

Finally we give an example of measures with $\bar{p}(\mu)=\frac{1}{2}$. The proposition is essentially known to hold (see Theorems 11.11 and 6.9 in [5]). However, Lemma 1.7 gives a very simple proof of this result.

Proposition 1.11. Let $\mu$ be a Radon probability measure on $I R^{n}$ and let $s<n$. If the set of points $a \in I R^{n}$ where

$$
0<\Theta_{*}^{s}(\mu, a):=\lim \inf _{r \downarrow 0} \frac{\mu(B(a, r))}{(2 r)^{s}} \leq \Theta^{* s}(\mu, a):=\lim \sup _{r \downarrow 0} \frac{\mu(B(a, r))}{(2 r)^{s}}<\infty
$$

holds has a positive $\mu$ measure then $\bar{p}(\mu)=\frac{1}{2}$.

Among the measures which this proposition applies to is the restriction of the $s$-dimensional Hausdorff measure $H^{s}$ to a $s$-dimensional self-similar set $E \subset \mathrm{IR}^{n}$ if $0<H^{s}(E)<\infty$ and $s<n$.

\subsection{Complementary results.}

We give other results related to very porous measures and to the doubling condition. The next lemma is used to characterize very porous measures in terms of a porosity property of their tangent measures. We denote by $U(x, r)$ the open ball centered at $x$ and with radius $r$.

Lemma 1.12. Let $\mu$ be a Radon probability measure on $\mathrm{IR}^{n}$, let $A \subset \mathrm{IR}^{n}$ and let $\alpha$ be a constant with $0<\alpha \leq \frac{1}{2}$. The following statement holds for $\mu$-a.e. $a \in A$. If $p(A, a) \geq \alpha$, then for every $\nu \in \operatorname{Tan}(\mu, a)$ there is a point $y \in B(0,1-\alpha)$ such that $\nu(U(y, \alpha))=0$.

From this lemma the following property follows.

Proposition 1.13. Let $\mu$ be a Radon probability measure on $I R^{n}$, let $\alpha$ be a constant with $0<\alpha \leq \frac{1}{2}$ and let

$C:=\left\{a \in \mathrm{IR}^{n}: \forall \nu \in \operatorname{Tan}(\mu, a)\right.$ there is an $y \in B(0,1-\alpha)$ such that $\left.\nu(U(y, \alpha))=0\right\}$. 
Then,

$$
\underline{p}(\mu)>\alpha \Longrightarrow \mu(C)>0
$$

and if $\mu$ satisfies the doubling condition $\mu$-a.e. then

$$
\mu(C)>0 \Longrightarrow \underline{p}(\mu) \geq \alpha .
$$

Finally, we state another property of measures which do not satisfy the doubling condition at a point $a \in \mathrm{IR}^{n}$. Given $A \subset \mathrm{IR}^{n}$, we denote by $\mu \mid A$ the restriction of the measure $\mu$ to the set $A$.

Proposition 1.14. Let $\mu$ be a Radon measure which does not satisfy the doubling condition at a point $a \in \mathrm{IR}^{n}$. Then, there is a sequence $\left\{r_{i}\right\} \downarrow 0$ such that the measures

$$
\frac{1}{\mu\left(B\left(a, r_{i}\right)\right)} T_{a, r_{i} \#}\left(\mu \mid B\left(a, r_{i}\right)\right)
$$

converge weakly to a probability measure on $\partial B(0,1)$.

\section{Proofs.}

\subsection{Proof of Theorem 1.2.}

\section{Proof of Lemma 1.1.}

It is easy to see that $\mu$ satisfies

$$
\lim \sup _{r \downarrow 0} \frac{\mu(B(x, r))}{\mu(B(x, \lambda r))}=\infty
$$

for all $\lambda \in(0,1)$ and all $x \in A$. Let $\left\{\lambda_{i}\right\}_{i \in \mathrm{IN}}$ be any sequence such that $\lim _{i \rightarrow \infty} \lambda_{i}=$ 1 with $0<\lambda_{i}<1$ for any $i \in \mathrm{IN}$. Given $\varepsilon>0$ and $x \in A$, by (2.1)

$$
\frac{\mu(B(x, r))}{\mu\left(B\left(x, \lambda_{i} r\right)\right)} \geq \frac{2^{i}}{\varepsilon}
$$

holds for arbitrarily small values of $r$. Let $\mathcal{V}_{i}$ be the Vitali class given by

$$
\mathcal{V}_{i}=\left\{B(x, r): x \in A, \frac{\mu(B(x, r))}{\mu\left(B\left(x, \lambda_{i} r\right)\right)} \geq \frac{2^{i}}{\varepsilon} \text { and } r<\frac{1}{i}\right\} .
$$


By Vitali covering theorem (see Theorem 2.8 in [5]), there is a sequence of disjointed balls $\left\{B_{i, j}\right\}_{j \in \mathrm{IN}} \subset \mathcal{V}_{i}, B_{i, j}=B\left(x_{i, j}, r_{i, j}\right)$, such that

$$
\mu\left(A \backslash \bigcup_{j=1}^{\infty} B_{i, j}\right)=0 .
$$

For all $i, j \in \mathrm{IN}$, let $B_{i, j}^{\prime}=B\left(x_{i, j}, \lambda_{i} r_{i, j}\right)$ and $W_{i, j}=B_{i, j} \backslash B_{i, j}^{\prime}$. Then

$$
\mu\left(B_{i, j}\right) \geq \frac{2^{i}}{\varepsilon} \mu\left(B_{i, j}^{\prime}\right)
$$

for all $i, j \in \mathrm{IN}$ which, together with (2.2), gives

$$
\begin{gathered}
\mu\left(A \backslash\left(\bigcap_{i=1}^{\infty} \bigcup_{j=1}^{\infty} W_{i, j}\right)\right)=\mu\left(\bigcup_{i=1}^{\infty}\left(A \backslash \bigcup_{j=1}^{\infty} W_{i, j}\right)\right) \leq \sum_{i=1}^{\infty} \mu\left(A \backslash \bigcup_{j=1}^{\infty} W_{i, j}\right)= \\
\sum_{i=1}^{\infty} \mu\left(A \bigcap \bigcup_{j=1}^{\infty} B_{i, j}^{\prime}\right) \leq \sum_{i=1}^{\infty} \frac{\varepsilon}{2^{i}} \mu\left(\bigcup_{j=1}^{\infty} B_{i, j}\right) \leq \varepsilon \mathbf{\square}
\end{gathered}
$$

\section{Proof of Theorem 1.2.}

i) For $\varepsilon>0$, let $C=\bigcap_{i=1}^{\infty} \bigcup_{j=1}^{\infty} W_{i, j}$ be the set used in Lemma 1.1 and $A^{*}=A \bigcap C$. Then $A^{*} \subset A$ and $\mu\left(A \backslash A^{*}\right) \leq \varepsilon$. We now check that $\bar{p}\left(A^{*}\right)=\frac{1}{2}$. If $x \in A^{*}$ then $x \in \bigcup_{j=1}^{\infty} W_{i, j}$ for all $i \in \mathrm{IN}$. Therefore, for all $i \in \mathrm{IN}$, there is a unique index $j(i)$ such that $x \in W_{i, j(i)}=B_{i, j(i)} \backslash B_{i, j(i)}^{\prime}$. Obviously $B_{i, j(i)}^{\prime} \subset B\left(x, 2 r_{i, j(i)}\right) \backslash A^{*}$ so that

$$
p\left(A^{*}, x, 2 r_{i, j(i)}\right) \geq \lambda_{i} r_{i, j(i)}
$$

holds for all $i \in \mathrm{IN}$. Consider the sequence of radius given by $\left\{2 r_{i, j(i)}\right\}_{i \in \mathrm{IN}}$. Since $r_{i, j(i)}$ is the radius of the ball $B_{i, j(i)}$ we have that $r_{i, j(i)}<\frac{1}{i}$ for all $i$, and by $(2.3)$ $\lim \sup _{i \rightarrow \infty} \frac{p\left(A^{*}, x, 2 r_{i, j(i)}\right)}{2 r_{i, j(i)}} \geq \frac{1}{2}$. Thus, $\lim \sup _{r \downarrow 0} \frac{p\left(A^{*}, x, r\right)}{r} \geq \frac{1}{2}$ and, since $\frac{p\left(A^{*}, x, r\right)}{r} \leq \frac{1}{2}$, the result follows.

ii) Let $A^{*}$ be as in part i) and let $A_{0}^{*}=A^{*}$. The argument used in Lemma 1.1 gives the existence of sets $A_{i}^{*} \subset A \backslash\left(\bigcup_{k=0}^{i-1} A_{k}^{*}\right), i \geq 1$ such that $\mu\left(A \backslash \bigcup_{k=0}^{i} A_{i}^{*}\right) \leq \varepsilon / 2^{i}$ and $\bar{p}\left(A_{i}^{*}\right)=\frac{1}{2}$. Thus the set $C=\bigcup_{i=0}^{\infty} A_{i}^{*} \subset A$ is a $\sigma$-strongly porous set and $\mu(C)=\lim _{i \rightarrow \infty} \mu\left(\bigcup_{k=0}^{i} A_{k}^{*}\right) \geq \mu(A)-\lim _{i \rightarrow \infty} \frac{\varepsilon}{2^{i}}=\mu(A)$.

\section{Proof of Corollary 1.4.}


The set $A^{*}$ of part (i) in Theorem 1.2 has a positive measure and its upper porosity is equal to $\frac{1}{2}$.

\section{Proof of Proposition 1.6.}

Let $A$ be the set of points where the doubling condition does not hold, let $\left\{\varepsilon_{j}\right\}$ be a sequence in $(0,1)$ such that $\lim _{j \rightarrow \infty} \varepsilon_{j}=0$, and let $x \in A$. Using (2.1) for $\lambda=1-\varepsilon_{j}$ we get that $\mu\left(B\left(x,\left(1-\varepsilon_{j}\right) r\right)\right) \leq \varepsilon_{j} \mu(B(x, r))$ holds for arbitrarily small values of $r$. Then $\operatorname{por}\left(\mu, x, r, \varepsilon_{j}\right) \geq\left(1-\varepsilon_{j}\right)$ for such values of $r$ and $\lim \sup _{r \rightarrow 0} \operatorname{por}\left(\mu, x, r, \varepsilon_{j}\right) \geq 1-\varepsilon_{j}$. Thus, $\lim _{j \rightarrow \infty} \limsup _{r \rightarrow 0} \operatorname{por}\left(\mu, x, r, \varepsilon_{j}\right) \geq 1$ and then $\overline{p o r}(\mu, x)=1$ for any $x \in A$. Therefore $\overline{p o r}(\mu)=1$.

\subsection{Proof of Theorem 1.8 .}

We first introduce results on tangent measures that we need later on. In [7] it is proved that if $\mu$ is an almost finite measure over $\operatorname{IR}^{n}$, then $\operatorname{Tan}(\mu, a) \neq \emptyset$ for $\mu$ almost every $a \in \mathrm{IR}^{n}$. If $\mu$ satisfies the doubling condition at $a$, then any sequence $\left\{r_{i}\right\} \downarrow 0$ contains a subsequence $\left\{r_{i_{j}}\right\}$ such that

$$
\frac{1}{\mu\left(B\left(a, r_{i_{j}}\right)\right)} T_{a, r_{i j}} \# \mu \stackrel{w}{\rightarrow} \nu \in \operatorname{Tan}(\mu, a)
$$

([5],Theorem 14.3). Furthermore, for all $\nu \in \operatorname{Tan}(\mu, a)$ there are a sequence $\left\{r_{i}\right\} \downarrow 0$ and a positive number $c$ such that $\nu=c \lim _{i \rightarrow \infty} \frac{1}{\mu\left(B\left(a, r_{i}\right)\right)} T_{a, r_{i} \#} \mu([5]$, Remark 14.4).

We denote by $\partial A$ the boundary of the set $A$. Recall that $U(x, r)$ is the open ball with center at $x \in \mathrm{IR}^{n}$ and radius $r$.

Lemma 2.1. Let $\mu$ be a Radon probability measure on $I R^{n}$, let $D$ be the set of points where the doubling condition holds and $A \subset D$. The following statement holds for $\mu$-a.e. $a \in A$.

If $\bar{p}(A, a)>0$, then there exist a $\nu^{*} \in \operatorname{Tan}(\mu, a)$ and an open half-space $H$ such that $0 \in \partial H$ and $\nu^{*}(H)=0$.

\section{Proof.}

Let $a \in A$ be a $\mu$-density point of $A$, that is

$$
\lim _{r \downarrow 0} \frac{\mu(B(a, r) \backslash A)}{\mu(B(a, r))}=0,
$$


let $\alpha=\bar{p}(A, a)>0$ and $0<\varepsilon<\alpha / 2$. We may select a sequence of radii $\left\{r_{i}\right\} \downarrow 0$ such that $p\left(A, a, r_{i}\right) \geq(\alpha-\varepsilon) r_{i}$ for all $i$ and $\frac{1}{\mu\left(B\left(a, r_{i}\right)\right)} T_{a, r_{i} \#} \mu \stackrel{w}{\rightarrow} \nu \in \operatorname{Tan}(\mu, a)$. Furthermore, since $p\left(A, a, r_{i}\right) \geq(\alpha-\varepsilon) r_{i}$, there is a sequence $\left\{z_{i}\right\}$ of points such that $B\left(z_{i},(\alpha-\varepsilon) r_{i}\right) \subset B\left(a, r_{i}\right) \backslash A$ for all $i$. Let $y_{i}=\frac{z_{i}-a}{r_{i}}$. By the compactness of $B(0,1-\alpha+\varepsilon)$, there is a subsequence of $\left\{y_{i}\right\}$, which for simplicity we also denote by $\left\{y_{i}\right\}$, such that $\lim _{i \rightarrow \infty} y_{i}=y \in B(0,1-\alpha+\varepsilon)$. Thus,

$$
\begin{aligned}
& \nu(U(y, \alpha-2 \varepsilon)) \leq \lim \inf _{i \rightarrow \infty} \frac{1}{\mu\left(B\left(a, r_{i}\right)\right)} T_{a, r_{i} \#} \mu(U(y, \alpha-2 \varepsilon)) \leq \\
& \lim _{i \rightarrow \infty} \frac{1}{\mu\left(B\left(a, r_{i}\right)\right)} T_{a, r_{i} \#} \mu\left(U\left(y_{i}, \alpha-\varepsilon\right)\right)=\lim \inf _{i \rightarrow \infty} \frac{1}{\mu\left(B\left(a, r_{i}\right)\right)} \mu\left(U\left(z_{i}, r_{i}(\alpha-\varepsilon)\right)\right) \\
& \leq \lim \inf _{i \rightarrow \infty} \frac{\mu\left(B\left(a, r_{i}\right) \backslash A\right)}{\mu\left(B\left(a, r_{i}\right)\right)}=0 .
\end{aligned}
$$

Thus $\operatorname{spt}(\nu) \neq \mathrm{IR}^{n}$ and there exists $\nu^{*} \in \operatorname{Tan}(\mu, a)$ and an open half space $H$ (see the proof of part (3) of Theorem 14.7 in [5]) such that $0 \in \partial H$, and $\nu^{*}(H)=0$.

Remark 1. This lemma was initially formulated stating that if $\bar{p}(A, a)=\alpha>0$, then there exist $y \in B(0,1-\alpha)$ and $\nu \in \operatorname{Tan}(\mu, a)$ such that $\nu(U(y, \alpha))=0$. The present formulation has been possible thanks to an anonymous referee who gave us the reference of Theorem 14.7 in [5]. This, together with Theorem 1.10, allowed us to obtain firstly that $\bar{p}(\mu)>0$ implies $\bar{p}(\mu) \geq \frac{1}{4}$, and afterwards we improved this result with Theorem 1.8.

\section{Proof of Lemma 1.7.}

We first prove that $\bar{p}(\mu)=\frac{1}{2} \Longrightarrow \mu(B)>0$.

If $\bar{p}(\mu)=\frac{1}{2}$ then for any $\varepsilon>0$ there is a set $E$ with $\mu(E)>0$ such that $\bar{p}(E)>$ $\frac{1}{2}-\varepsilon$. Then Lemma 2.1 gives $\mu(B) \geq \mu\left(E^{*}\right)=\mu(E)>0$ where $E^{*}=\{x \in E \cap D$ : there is $\nu \in \operatorname{Tan}(\mu, x)$ such that $\left.\operatorname{spt}(\nu) \neq \mathrm{IR}^{n}\right\}$.

We now prove that $\mu(B)>0 \Longrightarrow \bar{p}(\mu)=\frac{1}{2}$.

By Theorem 14.7 in [5], we know that for any $a \in B \cap D$ there are a measure $\nu^{*} \in \operatorname{Tan}(\mu, a)$ and an open half-space $H$ such that $0 \in \partial H$ and $\nu^{*}(H)=0$. Since $a \in D$ there exist a positive constant $c$ and a sequence $\left\{r_{i}\right\} \downarrow 0$ such that $\nu^{*}=$ $c_{\frac{1}{\mu\left(B\left(a, r_{i}\right)\right)}} \lim _{i \rightarrow \infty} T_{a, r_{i} \#} \mu$. Since $\nu^{*}(H)=0$, there exists a point $y \in H \cap \partial B\left(0, \frac{1}{2}\right)$ such that for any $\delta>0$

$$
\begin{aligned}
0 & =\nu^{*}\left(B\left(y, \frac{1}{2}-\delta\right)\right) \geq c \lim \sup _{i \rightarrow \infty} \frac{1}{\mu\left(B\left(a, r_{i}\right)\right)} T_{a, r_{i} \#} \mu\left(B\left(y, \frac{1}{2}-\delta\right)\right) \\
& =c \lim \sup _{i \rightarrow \infty} \frac{\mu\left(B\left(a+r_{i} y, r_{i}\left(\frac{1}{2}-\delta\right)\right)\right)}{\mu\left(B\left(a, r_{i}\right)\right)}
\end{aligned}
$$


holds. Thus, for any $\varepsilon>0, \frac{\mu\left(B\left(z_{i}, r_{i}\left(\frac{1}{2}-\delta\right)\right)\right)}{\mu\left(B\left(a, r_{i}\right)\right)}<\varepsilon$ holds for sufficiently large $i$, where $z_{i}:=a+r_{i} y$. Therefore for any $\delta, \varepsilon$ and $a \in B \cap D$, we have that $\operatorname{por}\left(\mu, a, r_{i}, \varepsilon\right) \geq$ $\frac{1}{2}-\delta$ for sufficiently large $i$. This implies (see 1.1) that $\overline{\operatorname{por}}(\mu) \geq \frac{1}{2}$. Since $\mu$ satisfies the doubling condition $\mu$-a.e. and $\bar{p}(\mu) \leq \frac{1}{2}$ we obtain $\frac{1}{2} \leq \overline{p o r}(\mu)=\bar{p}(\mu) \leq \frac{1}{2}$.

\section{Proof of Theorem 1.8.}

If $\mu$ does not satisfy the doubling condition $\mu$-a.e then Corollary 1.4 gives $\bar{p}(\mu)=\frac{1}{2}$.

Assume now that $\mu$ satisfies the doubling condition $\mu$-a.e. Let $\alpha$ be any constant with $0<\alpha<\bar{p}(\mu)$ and let $A$ be a set with $\mu(A)>0$ and $\bar{p}(A) \geq \alpha$. Using Lemma 2.1 we get that the set

$$
A^{*}:=\left\{a \in A: \text { there is } \nu \in \operatorname{Tan}(\mu, a) \text { such that } \operatorname{spt}(\nu) \neq \operatorname{IR}^{n}\right\}
$$

satisfies that $\mu\left(A^{*}\right)=\mu(A)>0$, and Lemma 1.7 gives the claim.

\section{Proof of Corollary 1.9.}

If $\mu$ satisfies the doubling condition $\mu$-a.e then $\bar{p}(\mu)=\overline{p o r}(\mu)$ and the above theorem gives that $\overline{\operatorname{por}}(\mu)$ only can take the values 0 or $\frac{1}{2}$. If $\mu$ does not satisfy the doubling condition $\mu$-a.e then Corollary 1.9 gives $\overline{\operatorname{por}}(\mu)=1$.

Notice that actually $\overline{p o r}(\mu)$ can take this three values: if $\mu$ does not satisfy the doubling $\mu$-a.e. then $\overline{\operatorname{por}}(\mu)=1$; if (1.3) holds $\mu$-a.e. then $\frac{1}{2}=\bar{p}(\mu)=\overline{\operatorname{por}}(\mu)$; and if the doubling condition holds and $\bar{p}(\mu)=0$ then $\overline{\operatorname{por}}(\mu)=0$.

\subsubsection{Proofs of Theorem 1.10 and Proposition 1.11.}

\section{Proof of Theorem 1.10.}

Since $\lambda:=\bar{p}(A)>0$, the set $B:=\{a \in A \cap D:$ there is $\nu \in \operatorname{Tan}(\mu, a)$ such that $\left.\operatorname{spt}(\nu) \neq \mathrm{IR}^{n}\right\}$ satisfies $\mu(B)=\mu(A)$ (see Lemma 2.1). We now prove that for any $\varepsilon, 0<\varepsilon<\mu(A)$, there exists a set $A^{*} \subset B$ such that $\mu\left(B \backslash A^{*}\right) \leq \varepsilon$ and $\bar{p}\left(A^{*}\right) \geq \frac{1}{4}$. Since $\mu(B)=\mu(A)$ this gives the claim.

Let $a \in B$ and $\nu \in \operatorname{Tan}(\mu, a)$ such that $\operatorname{spt}(\nu) \neq \mathrm{IR}^{n}$. Then, there exists $\nu^{*} \in \operatorname{Tan}(\mu, a)$ and an open half-space $H$ such that $0 \in \partial H$ and $\nu^{*}(H)=0$. Since $a \in D$, there exist a positive constant $c$ and a sequence $\left\{r_{i}\right\} \downarrow 0$ such that $\nu^{*}=c \lim _{i \rightarrow \infty} \frac{1}{\mu\left(B\left(a, r_{i}\right)\right)} T_{a, r_{i} \#} \mu$. Since $\nu^{*}(H)=0$, there is a point $y \in H \cap \partial B(0,1 / 2)$ 
such that for any $\delta>0$

$$
\begin{aligned}
0 & =\nu^{*}\left(B\left(y, \frac{1}{2}-\delta\right)\right) \geq c \lim \sup _{i \rightarrow \infty} \frac{1}{\mu\left(B\left(a, r_{i}\right)\right)} T_{a, r_{i} \#} \mu\left(B\left(y, \frac{1}{2}-\delta\right)\right) \\
& =c \lim \sup _{i \rightarrow \infty} \frac{\mu\left(B\left(a+r_{i} y, r_{i}\left(\frac{1}{2}-\delta\right)\right)\right)}{\mu\left(B\left(a, r_{i}\right)\right)}
\end{aligned}
$$

holds. Then, given an $\varepsilon>0$ and a $k>0$, there is an $i_{k}$ such that

$$
\frac{\mu\left(B\left(a+r_{i} y, r_{i}\left(\frac{1}{2}-2^{-k}\right)\right)\right)}{\mu\left(B\left(a, r_{i}\right)\right)}<\frac{\varepsilon}{2^{k}} \text { for } i>i_{k} .
$$

Let $\mathcal{V}_{k}$ be the Vitali class given by

$$
\begin{aligned}
\mathcal{V}_{k}=\{B(a, r): \quad & a \in B, r<\frac{1}{k} \text { and there is an } y \in \partial B(0,1 / 2) \text { such that } \\
& \left.\frac{\mu\left(B\left(a+r y, r\left(\frac{1}{2}-2^{-k}\right)\right)\right)}{\mu(B(a, r))}<\frac{\varepsilon}{2^{k}}\right\} .
\end{aligned}
$$

By Vitali covering theorem, there is a sequence of disjointed balls $\left\{B_{k, j}\right\}_{j=1}^{\infty} \subset \mathcal{V}_{k}$, $B_{k, j}=B\left(x_{k, j}, r_{k, j}\right)$, satisfying

$$
\mu\left(B \backslash \bigcup_{j=1}^{\infty} B_{k, j}\right)=0 .
$$

Since each ball $B_{k, j} \in \mathcal{V}_{k}$, there is an $y_{k, j} \in \partial B\left(0, \frac{1}{2}\right)$ such that

$$
\frac{\mu\left(B_{k, j}^{\prime}\right)}{\mu\left(B_{k, j}\right)}<\frac{\varepsilon}{2^{k}}
$$

where $B_{k, j}^{\prime}=B\left(x_{k, j}+r_{k, j} y_{k, j},\left(\frac{1}{2}-2^{-k}\right) r_{k, j}\right)$. Let $W_{k, j}=B_{k, j} \backslash B_{k, j}^{\prime}$ and $A^{*}=$ $B \cap\left(\bigcap_{k=1}^{\infty} \bigcup_{j=1}^{\infty} W_{k, j}\right)$. Using (2.4) and (2.5) we obtain $\mu\left(A^{*}\right)>\mu(B)-\varepsilon=$ $\mu(A)-\varepsilon$. Let $x \in A^{*}$, then for all $k \in \mathrm{IN}, x \in \bigcup_{j=1}^{\infty} W_{k, j}$ holds. Thus, there is an unique index $j(k)$ such that $x \in W_{k, j(k)}$. Since $B_{k, j(k)}^{\prime} \subset B\left(x, 2 r_{k, j(k)}\right) \backslash A^{*}$ we have that $p\left(A^{*}, x, 2 r_{k, j(k)}\right) \geq\left(\frac{1}{2}-2^{-k}\right) r_{k, j(k)}$ and then $\bar{p}\left(A^{*}, x\right) \geq \frac{1}{4}$ for all $x \in A^{*}$.

Remark 2. Let $D$ be the set of points where the doubling condition holds. If $\mu(D)<1$ then, for any $\varepsilon, 0<\varepsilon<\mu\left(A \cap D^{c}\right)$, there is a set $A^{*} \subset A \cap D^{c}$ such that $\mu\left(A^{*}\right) \geq \mu\left(A \cap D^{c}\right)-\varepsilon$ and $\bar{p}\left(A^{*}\right)=\frac{1}{2}$. 


\section{Proof of Proposition 1.11.}

Let $D \supset A$ be the set of points where the doubling condition holds. Theorem 14.7 in [5] guarantees that for $\mu$-a.e. $a \in A$ and every $\nu \in \operatorname{Tan}(\mu, a)$, there is a positive number $c$ such that

$$
t c r^{s} \leq \nu(B(x, r)) \leq c r^{s}, \text { for } x \in \operatorname{spt}(\nu), 0<r<\infty,
$$

where $t=t(a)=\Theta_{*}^{s}(\mu, a) / \Theta^{* s}(\mu, a)$. Therefore, since $s<n$ we have that $\operatorname{spt}(\nu) \neq$ $\operatorname{IR}^{n}$ for every $\nu \in \operatorname{Tan}(\mu, a)$ and $\mu$-a.e. $a \in A$ (see [5], Chap. 14, exer. 4). Thus the set $A_{1}=\left\{a \in A\right.$ : there exists $\nu \in \operatorname{Tan}(\mu, a)$ such that $\left.\operatorname{spt}(\nu) \neq \mathrm{IR}^{n}\right\}$ satisfies that $\mu\left(A_{1}\right)=\mu(A)>0$, and Lemma 1.7 gives $\bar{p}(\mu)=\frac{1}{2}$ provided $\mu(D)=1$. If $\mu(D)<1$ then Corollary 1.4 gives the result.

\subsection{Proofs of complementary results.}

\section{Proof of Lemma 1.12.}

Let $a$ be a $\mu$-density point of $A$, that is

$$
\lim _{r \downarrow 0} \frac{\mu(B(a, r) \backslash A)}{\mu(B(a, r))}=0,
$$

and let $\nu=\lim _{i \rightarrow \infty} c_{i} T_{a, r_{i} \#} \mu \in \operatorname{Tan}(\mu, a)$. Then (see Remark 14.4, part (1), in [5]) there are a subsequence $\left\{r_{i_{j}}\right\}$ of $\left\{r_{i}\right\}$ and a constant $R>1$ such that $\nu=\lim _{j \rightarrow \infty} \frac{c}{\mu\left(B\left(a, R r_{i_{j}}\right)\right)} T_{a, r_{i_{j}}} \# \mu$. Let $\left\{\varepsilon_{k}\right\}$ be a decreasing sequence tending to zero. Since $\underline{p}(A, a) \geq \alpha$, for a given $\varepsilon_{k}$, there is an $i_{k}$ such that $p\left(A, a, r_{i_{j}}\right) \geq\left(\alpha-\varepsilon_{k}\right) r_{i_{j}}$ for all $\bar{i}_{j}>i_{k}$. The argument used in Lemma 2.1 gives a point $y_{k} \in B\left(0,1-\alpha+\varepsilon_{k}\right)$ such that

$$
\nu\left(U\left(y_{k}, \alpha-2 \varepsilon_{k}\right)\right) \leq c \lim \inf _{j \rightarrow \infty} \frac{\mu\left(B\left(a, r_{i_{j}}\right) \backslash A\right)}{\mu\left(B\left(a, R r_{i_{j}}\right)\right)} \leq c \lim \inf _{j \rightarrow \infty} \frac{\mu\left(B\left(a, r_{i_{j}}\right) \backslash A\right)}{\mu\left(B\left(a, r_{i_{j}}\right)\right)}=0 .
$$

The sequence $\left\{y_{k}\right\}$ has a subsequence which converges to a point $y \in B(0, \alpha)$. Let $\delta>0$. There is an index $k$ such that

$$
\nu(U(y, \alpha-\delta)) \leq \nu\left(U\left(y_{k}, \alpha-2 \varepsilon_{k}\right)\right)=0
$$

and letting $\delta \downarrow 0$ the claim follows.

\section{Proof of Proposition 1.13.}


We first prove $\underline{p}(\mu)>\alpha \Longrightarrow \mu(C)>0$.

Since $\underline{p}(\mu)>\alpha$ there is a set $E$ with $\mu(E)>0$ such that $\underline{p}(E) \geq \alpha$. Lemma 1.12 gives that the set $E^{*}=\{a \in E$ : for any $\nu \in \operatorname{Tan}(\mu, a)$ there exists $y \in B(0,1-\alpha)$ such that $\nu(U(y, \alpha))=0\}$ satisfies that $\mu\left(E^{*}\right)=\mu(E)>0$ so that $\mu(C)>0$.

We now prove $\mu(C)>0 \Longrightarrow p(\mu) \geq \alpha$.

Let $D$ be the set of points where the doubling condition holds. Since $\mu(D)=1$ then $p(\mu)=\operatorname{por}(\mu)$ holds (see 1.2). Then, it is sufficient to prove that for any $x \in \bar{C} \cap D$ and $\varepsilon>0$,

$$
\lim \inf _{r \downarrow 0} \operatorname{por}(\mu, x, r, \varepsilon) \geq \alpha .
$$

If this is not the case, there are $x \in C \cap D, \varepsilon>0$, a sequence of radii $\left\{r_{i}\right\} \downarrow 0$ such that

$$
\operatorname{por}\left(\mu, x, r_{i}, \varepsilon\right)<\frac{p+\alpha}{2}
$$

where $p:=\liminf _{r \downarrow 0} \operatorname{por}(\mu, x, r, \varepsilon)$. Since $x \in D$ there exist a subsequence $\left\{r_{i_{j}}\right\}$ of $\left\{r_{i}\right\}$ and a point $y \in B(0,1-\alpha)$ such that $\frac{1}{\mu\left(B\left(x, r_{i_{j}}\right)\right)} T_{x, r_{i_{j}} \# \mu} \stackrel{w}{\rightarrow} \nu \in \operatorname{Tan}(\mu, x)$ and $\nu(U(y, \alpha))=0$. Let $\delta$ be a constant with $0<\delta<(\alpha-p) / 2$. Then,

$$
0=\nu(B(y, \alpha-\delta)) \geq \lim \sup _{i \rightarrow \infty} \frac{\mu\left(B\left(x+r_{i_{j}} y, r_{i_{j}}(\alpha-\delta)\right)\right.}{\mu\left(B\left(x, r_{i_{j}}\right)\right)}
$$

holds. Hence for any $\varepsilon>0$ there are $j_{0}$ and $z_{j}:=x+r_{i_{j}} y$ such that $\mu\left(B\left(z_{j}, r_{i_{j}}(\alpha-\right.\right.$ $\delta)) \leq \varepsilon \mu\left(B\left(x, r_{i_{j}}\right)\right)$ and $B\left(z_{j}, r_{i_{j}}(\alpha-\delta)\right) \subset B\left(x, r_{i_{j}}\right)$ for $j>j_{0}$. Therefore $\operatorname{por}\left(\mu, x, r_{i_{j}}, \varepsilon\right) \geq$ $\alpha-\delta>\frac{p+\alpha}{2}$ which contradicts (2.6).

\section{Proof of Proposition 1.14.}

For $i \in \mathrm{IN}$, let $\lambda_{i}=1-2^{-i}$. Since $\mu$ does not satisfy the doubling condition at $a$, it follows that

$$
\frac{\mu(B(a, r))}{\mu\left(B\left(a, \lambda_{i} r\right)\right)}>2^{i}
$$

for arbitrarily small values of $r$. Thus, we may select a sequence $\left\{r_{j}\right\} \downarrow 0$ such that $\mu\left(B\left(a, r_{j}\right)\right)>2^{j} \mu\left(B\left(a, \lambda_{j} r_{j}\right)\right)$. Let $\left\{\nu_{j}\right\}$ be the sequence of measures given by $\nu_{j}=\frac{1}{\mu\left(B\left(a, r_{j}\right)\right)} T_{a, r_{j} \#}\left(\mu \mid B\left(a, r_{j}\right)\right)$ and take $R>0$. Then,

$$
\nu_{j}(B(0, R))=\frac{\mu\left(B\left(a, r_{j}\right) \cap B\left(a, R r_{j}\right)\right)}{\mu\left(B\left(a, r_{j}\right)\right)} \leq 1,
$$

and $\sup \left\{\nu_{j}(K): j=1,2, \ldots\right\}<\infty$ for all compact sets $K \subset \mathrm{IR}^{n}$. Therefore there is a subsequence $\left\{\nu_{j_{k}}\right\}$ of $\left\{\nu_{j}\right\}$, which converges weakly to some measure 
$\nu$. It is easy to see that $\nu$ is a probability measure on $B(0,1)$. We now see that $\nu(\partial B(0,1))=1$. Let $C_{i}=B(0,1) \backslash U\left(0, \lambda_{i}\right)$, then

$$
\begin{aligned}
& \nu_{j_{k}}\left(C_{i}\right)=\frac{\mu\left(B\left(a, r_{j_{k}}\right) \backslash U\left(a, \lambda_{i} r_{j_{k}}\right)\right)}{\mu\left(B\left(a, r_{j_{k}}\right)\right)} \geq \frac{\mu\left(B\left(a, r_{j_{k}}\right) \backslash U\left(a, \lambda_{j_{k}} r_{j_{k}}\right)\right)}{\mu\left(B\left(a, r_{j_{k}}\right)\right)}>1-2^{-k} \text { for } j_{k}>i, \\
& \text { so } \nu\left(C_{i}\right) \geq \lim \sup _{k \rightarrow \infty} \nu_{j_{k}}\left(C_{i}\right) \geq 1, \text { and we get } \nu(\partial B(0,1))=\lim _{i \rightarrow \infty} \nu\left(C_{i}\right)=1 \text {. }
\end{aligned}
$$

\section{Acknowledgments.}

An anonymous referee has significantly contributed to the improvement of the results of this paper (see Remark 1). We are grateful to the helpful remarks of Pertti Mattila and Esa and Maarit Järvenpää. This research was partially supported by the Dirección General de Enseñanza Superior e Investigación. PB970301.

\section{FINAL REMARK}

At the time of revising the galley proofs of this paper we have known that Theorem 1.8 can also be proved using results of Luděk Zajiček (see [11]). These results also allows us to prove that Theorem 1.10 holds with $\bar{p}\left(A^{*}\right)>c$ for $c$ arbitrarily close to $\frac{1}{2}$ (see $\left.[6]\right)$.

\section{References}

[1] R. R. Coifmann and G. Weiss, Analyse Harmonique Non-commutative sur Certains Espaces Homogènes, Lectures Notes in Math. vol. 242 (1971), Springer-Verlag.

[2] H. Federer, Geometric Measure Theory (1969), Springer Verlag.

[3] J-P. Eckmann, E. Järvenpää and M. Järvenpää, Porosities and Dimensions of Measures, Nonlinearity 13 (2000), 1-18.

[4] M. de Guzmán, Differentiation of Integrals in $\mathrm{IR}^{n}$, Lectures Notes in Math. vol. 481 (1975), Springer-Verlag.

[5] P. Mattila, Geometry of sets and measures in Euclidean spaces (1995), Cambridge University Press.

[6] M. Eugenia Mera and Manuel Morán. Upper Porosities of Measures and Sets. Manuscript in preparation. 
[7] D. Preiss, Geometry of measures in $\mathrm{IR}^{n}$, Ann. of Math., (2) 125 (1987), 537-643.

[8] A. Salli, On the Minkowski dimension of strongly porous fractal sets in $\mathrm{IR}^{n}$, Proc. London Math. Soc, (3) 62 (1991), 353-372.

[9] B. S. Thomson, Real Functions, Lectures Notes in Math, vol. 1170 (1985), Springer-Verlag.

[10] L. Zajiček, Porosity and $\sigma$-porosity, Real Analysis Exchange, 13 (1987-88), 314-350.

[11] L. Zajiček. Sets of $\sigma$-porosity and sets of $\sigma$-porosity $(q)$. Časopis Pěst. Mat. 101 (1976), 350-359.

M. Eugenia Mera, Manuel Morán.

Departamento de Análisis Económico.Universidad Complutense.

Campus de Somosaguas. 28223 Madrid. España.

E-Mail Address: ececo06@sis.ucm.es 